\title{
Super Congruences Involving Alternating Harmonic Sums
}

\author{
Zhongyan Shen ${ }^{1}$, Tianxin $\mathrm{Cai}^{2}$ \\ ${ }^{1}$ Department of Mathematics, Zhejiang International Studies University, Hangzhou, China \\ ${ }^{2}$ Department of Mathematics, Zhejiang University, Hangzhou, China \\ Email: huanchenszyan@163.com,txcai@zju.edu.cn
}

How to cite this paper: Shen, Z.Y. and Cai, T.X. (2020) Super Congruences Involving Alternating Harmonic Sums. Advances in Pure Mathematics, 10, 611-622. https://doi.org/10.4236/apm.2020.1010037

Received: September 23, 2020

Accepted: October 26, 2020

Published: October 29, 2020

Copyright $\odot 2020$ by author(s) and Scientific Research Publishing Inc. This work is licensed under the Creative Commons Attribution International License (CC BY 4.0).

http://creativecommons.org/licenses/by/4.0/

\section{(c) (i) Open Access}

\section{Abstract}

Let $p$ be an odd prime, the harmonic congruence such as

$$
\sum_{i+j+k=p} \frac{1}{i j k} \equiv-2 B_{p-3}(\bmod p),
$$

and many different variations and generalizations have been studied intensively. In this note, we consider the congruences involving the combination of alternating harmonic sums,

$$
\sum_{\substack{i_{1}+i_{2}+\cdots+i_{n}=p^{r} \\ i_{1}, i_{2}, \cdots, i_{n} \in \mathcal{P}_{p}}} \frac{(-1)^{i_{1}}}{i_{1} i_{2} \cdots i_{n}}, \sum_{\substack{i_{1}+i_{2}+\cdots+i_{n}=p^{r} \\ i_{1}, i_{2}, \cdots, i_{n} \in \mathcal{P}_{p}}} \frac{(-1)^{i_{1} i_{2}}}{i_{1} i_{2} \cdots i_{n}}, \cdots, \sum_{\substack{i_{1}+i_{2}+\cdots+i_{n}=p^{r} \\ i_{1}, i_{2}, \cdots, i_{n} \in \mathcal{P}_{p}}} \frac{(-1)^{i_{1}+i_{2}+\cdots+i_{n}}\left[\frac{n}{2}\right]}{i_{1} i_{2} \cdots i_{n}} .
$$

where $\mathcal{P}_{p}$ denotes the set of positive integers which are prime to $p$. And we establish the combinational congruences involving alternating harmonic sums for positive integer $n=3,4,5$.

\section{Keywords}

Bernoulli Numbers, Alternating Harmonic Sums, Congruences, Modulo Prime Powers

\section{Introduction}

At the beginning of the $21^{\text {th }}$ century, Zhao (Cf. [1]) first announced the following curious congruence involving multiple harmonic sums for any odd prime $p>3$,

$$
\sum_{i+j+k=p} \frac{1}{i j k} \equiv-2 B_{p-3}(\bmod p),
$$

which holds when $p=3$ evidently. Here, Bernoulli numbers $B_{k}$ are defined by the recursive relation: 


$$
\sum_{i=0}^{n}\left(\begin{array}{c}
n+1 \\
i
\end{array}\right) B_{i}=0, n \geq 1 .
$$

A simple proof of (1) was presented in [2]. This congruence has been generalized along several directions. First, Zhou and Cai [3] established the following harmonic congruence for prime $p>3$ and integer $n \leq p-2$

$$
\sum_{l_{1}+l_{2}+\cdots+l_{n}=p} \frac{1}{l_{1} l_{2} \cdots l_{n}} \equiv\left\{\begin{array}{l}
-(n-1) ! B_{p-n}(\bmod p), \text { if } 2 \nmid n, \\
-\frac{n(n !)}{2(n+1)} p B_{p-n-1}\left(\bmod p^{2}\right), \text { if } 2 \mid n .
\end{array}\right.
$$

Later, Xia and Cai [4] generalized (1) to

$$
\sum_{i+j+k=p} \frac{1}{i j k} \equiv \frac{12 B_{p-3}}{p-3}-\frac{3 B_{2 p-4}}{p-4}\left(\bmod p^{2}\right),
$$

where $p>5$ is a prime.

Recently, Wang and Cai [5] proved for every prime $p \geq 3$ and positive integer $r$

$$
\sum_{\substack{i+j+k=p^{r} \\ i, j, k \in \mathcal{P}_{p}}} \frac{1}{i j k} \equiv-2 p^{r-1} B_{p-3}\left(\bmod p^{r}\right),
$$

where $\mathcal{P}_{p}$ denotes the set of positive integers which are prime to $p$.

Let $n=2$ or 4 , for every positive integer $r \geq \frac{n}{2}$ and prime $p>n$, Zhao [6] extended (3) to

$$
\sum_{\substack{i_{1}+i_{2}+\cdots+i_{n}=p^{r} \\ i_{1}, i_{2}, \cdots, i_{n} \in \mathcal{P}_{p}}} \frac{1}{i_{1} i_{2} \cdots i_{n}} \equiv-\frac{n !}{n+1} p^{r} B_{p-n-1}\left(\bmod p^{r+1}\right)
$$

For any prime $p>5$ and integer $r>1$, Wang [7] proved that

$$
\sum_{\substack{i_{1}+i_{2}+\cdots+i_{5}=p^{r} \\ i_{1}, i_{2}, \cdots, i_{5} \in \mathcal{P}_{p}}} \frac{1}{i_{1} i_{2} \cdots i_{5}} \equiv-\frac{5 !}{6} p^{r-1} B_{p-5}\left(\bmod p^{r}\right) .
$$

We consider the following alternating harmonic sums

$$
\sum_{\substack{i_{1}+i_{2}+\cdots+i_{n}=p^{r} \\ i_{1}, i_{2}, \cdots, i_{n} \in \mathcal{P}_{p}}} \frac{\sigma_{1}^{i_{1}} \sigma_{2}^{i_{2}} \cdots \sigma_{n}^{i_{n}}}{i_{1} i_{2} \cdots i_{n}}
$$

where $\sigma_{i} \in\{1,-1\}, i=1,2, \cdots, n$. Given $n$, we only need to consider the following alternating harmonic sums,

$$
\sum_{\substack{i_{1}+i_{2}+\cdots+i_{n}=p^{r} \\ i_{1}, i_{2}, \cdots, i_{n} \in \mathcal{P}_{p}}} \frac{(-1)^{i_{1}}}{i_{1} i_{2} \cdots i_{n}}, \sum_{\substack{i_{1}+i_{2}+\cdots+i_{n}=p^{r} \\ i_{1}, i_{2}, \cdots, i_{n} \in \mathcal{P}_{p}}} \frac{(-1)^{i_{1}+i_{2}}}{i_{1} i_{2} \cdots i_{n}}, \cdots, \sum_{\substack{i_{1}+i_{2}+\cdots+i_{n}=p^{r} \\ i_{1}, i_{2}, \cdots, i_{n} \in \mathcal{P}_{p}}} \frac{(-1)^{i_{1}+i_{2}+\cdots+i_{n}}\left[\frac{n}{2}\right]}{i_{1} i_{2} \cdots i_{n}}
$$

where $[x]$ denotes the largest integer less than or equal to $x$.

In this paper, we consider the congruences involving the combination of alternating harmonic sums, 


$$
\sum_{\substack{i_{1}+i_{2}+\cdots+i_{n}=p^{r} \\ i_{1}, i_{2}, \cdots, i_{n} \in \mathcal{P}_{p}}} \frac{(-1)^{r}}{i_{1} i_{2} \cdots i_{n}}, \sum_{\substack{i_{1} \\ i_{1}+i_{2}+\cdots+i_{2}=p^{r} \\ i_{1}, i_{2}, \cdots, i_{n} \in \mathcal{P}_{p}}} \frac{(-1)^{i_{1}+i_{2}}}{i_{1} i_{2} \cdots i_{n}}, \cdots, \sum_{\substack{i_{1}+i_{2}+\cdots+i_{n}=p^{r} \\ i_{1}, i_{2}, \cdots, i_{n} \in \mathcal{P}_{p}}} \frac{(-1)^{i_{1}+i_{2}+\cdots+i_{n}}\left[\frac{n}{2}\right]}{i_{1} i_{2} \cdots i_{n}} .
$$

We obtain the following theorems. Among them, Theorem 1 and Theorem 2 have been proved by Wang [8] using different method.

Theorem 1. Let $p$ be an odd prime and $r$ a positive integer, then

$$
\sum_{\substack{i+j+k=2 p^{r} \\ i, j, k \in \mathcal{P}_{p}}} \frac{(-1)^{i}}{i j k} \equiv p^{r-1} B_{p-3}\left(\bmod p^{r}\right)
$$

Remark 1. There is no solution $(i, j, k)$ for the equation $i+j+k=2 p^{r}$ with $i, j, k \in \mathcal{P}_{2 p}$.

Theorem 2. Let $p$ be an odd prime and $r$ a positive integer, then

$$
\sum_{\substack{i+j+k=p^{r} \\ i, j, k \in \mathcal{P}_{p}}} \frac{(-1)^{i}}{i j k} \equiv \frac{1}{2} p^{r-1} B_{p-3}\left(\bmod p^{r}\right) .
$$

Theorem 3. Let $p \geq 5$ be a prime and $r$ a positive integer, then

$$
\begin{aligned}
& 4 \sum_{\substack{i_{1}+i_{2}+i_{3}+i_{4}=2 p^{r} \\
i_{1}, i_{2}, i_{3}, i_{4} \in \mathcal{P}_{p}}} \frac{(-1)^{i_{1}}}{i_{1} i_{2} i_{3} i_{4}}+3 \sum_{\substack{i_{1}+i_{2}+i_{3}+i_{4}=2 p^{r} \\
i_{1}, i_{2}, i_{3}, i_{4} \in \mathcal{P}_{p}}} \frac{(-1)^{i_{1}+i_{2}}}{i_{1} i_{2} i_{3} i_{4}} \\
& \equiv\left\{\begin{array}{l}
\frac{216}{5} p B_{p-5}\left(\bmod p^{2}\right), \text { if } r=1, \\
\frac{36}{5} p^{r} B_{p-5}\left(\bmod p^{r+1}\right), \text { if } r>1 .
\end{array}\right.
\end{aligned}
$$

Theorem 4. Let $p>5$ be a prime and $r$ a positive integer, then

$$
\begin{aligned}
& \sum_{\substack{i_{i}+i_{2}+i_{3}+i_{i}+i_{i}=2 p^{r} \\
i_{1}, i_{2}, i_{3}, i_{4}, i_{5} \in \mathcal{P}_{p}}} \frac{(-1)^{r}}{i_{1} i_{2} i_{3} i_{4} i_{5}}+2 \sum_{\substack{i_{1} \\
i_{1}+i_{2}+i_{i}+i_{4}+i_{5}=2 p^{r} \\
i_{1}, i_{2}, i_{3}, i_{4}, i_{5} \in \mathcal{P}_{p}}} \frac{(-1)^{r}}{i_{1} i_{2} i_{3} i_{4} i_{4} i_{5}} \\
& \equiv\left\{\begin{array}{l}
12 B_{p-5}(\bmod p), \text { if } r=1, \\
6 p^{r-1} B_{p-5}\left(\bmod p^{r}\right), \text { if } r>1 .
\end{array}\right.
\end{aligned}
$$

\section{Preliminaries}

In order to prove the theorems, we need the following lemmas.

Lemma 1 ([5]) Let $p$ be an odd prime and $r, m$ positive integers, then

$$
\sum_{\substack{i+j+k=m p \\ i, j, k \in \mathcal{P}_{p}}} \frac{1}{i j k} \equiv-2 m p^{r-1} B_{p-3}\left(\bmod p^{r}\right) .
$$

Lemma 2. Let $p$ be an odd prime and $r$, m positive integers, then

$$
\sum_{\substack{i+j+k=m p^{r} \\ i, j, k \in \mathcal{P}_{p}}} \frac{1}{i j k}=\frac{6}{m p^{r}} \sum_{\substack{1 \leq j<l \leq m p^{r} \\ j, l, l-j \in \mathcal{P}_{p}}} \frac{1}{j l} .
$$

Proof. It is easy to see that 


$$
\sum_{\substack{i+j+k=m p^{r} \\ i, j, k \in \mathcal{P}_{p}}} \frac{1}{i j k}=\frac{1}{m p^{r}} \sum_{\substack{i+j+k=m p^{r} \\ i, j, k \in \mathcal{P}_{p}}} \frac{i+j+k}{i j k}=\frac{3}{m p^{r}} \sum_{\substack{i+j+k=m p^{r} \\ i, j, k \in \mathcal{P} p}} \frac{1}{i j} .
$$

Let $l=j+k$, then $1 \leq j<l \leq m p^{r}$ and $j, l, l-j \in \mathcal{P}_{p}$. By symmetry, we have

$$
\frac{3}{m p^{r}} \sum_{\substack{i+j+k=m p^{r} \\ i, j, k \in \mathcal{P}_{p}}} \frac{1}{i j}=\frac{3}{m p^{r}} \sum_{\substack{i+j<m p^{r} \\ i, j, l \in \mathcal{P}_{p}}} \frac{1}{l} \frac{i+j}{i j}=\frac{6}{m p^{r}} \sum_{\substack{1 \leq j<l \leq m p^{r} \\ j, l, l-j \in \mathcal{P}_{p}}} \frac{1}{j l} .
$$

This completes the proof of Lemma 2.

Lemma 3. Let $p>4$ be a prime and $r, m$ positive integers, then

$$
\sum_{\substack{i_{1}+i_{2}+i_{3}+i_{4}=m p^{r} \\ i_{1}, i_{2}, i_{3}, i_{4} \in \mathcal{P}_{p}}} \frac{1}{i_{1} i_{2} i_{3} i_{4}}=\frac{24}{m p^{r}} \sum_{\substack{1 \leq u_{1}<u_{2}<u_{3} \leq m p^{r} \\ u_{1}, u_{3}, u_{2}-u_{1}, u_{3}-u_{2} \in \mathcal{P}_{p}}} \frac{1}{u_{1} u_{2} u_{3}} .
$$

Proof. The proof of Lemma 3 is similar to the proof of Lemma 2.

Lemma 4 ([3]) Let $r, \alpha_{1}, \cdots, \alpha_{n}$ be positive integers, $r=\alpha_{1}+\cdots+\alpha_{n} \leq p-3$, then

$$
\sum_{\substack{1 \leq l_{1}, \cdots, l_{n} \leq p-1 \\
l_{i} \neq l_{j}, \forall i \neq j}} \frac{1}{l_{1}^{\alpha_{1}} l_{2}^{\alpha_{2}} \cdots l_{n}^{\alpha_{n}}} \equiv\left\{\begin{array}{l}
(-1)^{n}(n-1) ! \frac{r(r+1)}{2(r+2)} B_{p-r-2} p^{2}\left(\bmod p^{3}\right), \text { if } 2 \nmid r, \\
(-1)^{n-1}(n-1) ! \frac{r}{r+1} B_{p-r-1} p\left(\bmod p^{2}\right), \text { if } 2 \mid r .
\end{array}\right.
$$

Lemma 5 ([7]). Let $p$ be an odd prime, and $\alpha_{1}, \cdots, \alpha_{n}$ positive integers, where $r=\alpha_{1}+\cdots+\alpha_{n} \leq p-3$, then

$$
\sum_{\substack{1 \leq l_{1}, \cdots, l_{n} \leq 2 p \\
l_{i} \neq l_{j}, l_{i} \in \mathcal{P}_{p}}} \frac{1}{l_{1}^{\alpha_{1}} l_{2}^{\alpha_{2}} \cdots l_{n}^{\alpha_{n}}} \equiv\left\{\begin{array}{l}
(-1)^{n}(n-1) ! \frac{2 r(r+1)}{r+2} B_{p-r-2} p^{2}\left(\bmod p^{3}\right), \text { if } 2 \nmid r, \\
(-1)^{n-1}(n-1) ! \frac{2 r}{r+1} B_{p-r-1} p\left(\bmod p^{2}\right), \text { if } 2 \mid r .
\end{array}\right.
$$

Lemma 6. Let $p>4$ be a prime, then

$$
\sum_{\substack{i_{1}+i_{2}+i_{3}+i_{4}=2 p \\ i_{1}, i_{2}, i_{3}, i_{4} \in \mathcal{P}_{p}}} \frac{1}{i_{1} i_{2} i_{3} i_{4}} \equiv-\frac{240}{5} p B_{p-5}\left(\bmod p^{2}\right) .
$$

Proof. By Lemma 3, we have

$$
\sum_{\substack{i_{1}+i_{2}+i_{3}+i_{4}=2 p \\ i_{1}, i_{2}, i_{3}, i_{4} \in \mathcal{P}_{p}}} \frac{1}{i_{1} i_{2} i_{3} i_{4}}=\frac{24}{2 p} \sum_{\substack{1 \leq u_{1}<u_{2}<u_{3} \leq 2 p \\ u_{1}, u_{3}, u_{2}-u_{1}, u_{3}-u_{2} \in \mathcal{P}_{p}}} \frac{1}{u_{1} u_{2} u_{3}} .
$$

It is easy to see that

$$
\sum_{\substack{1 \leq u_{1}<u_{2}<u_{3} \leq 2 p \\ u_{1}, u_{3}, u_{2}-u_{1}, u_{3}-u_{2} \in \mathcal{P}_{p}}} \frac{1}{u_{1} u_{2} u_{3}}=\sum_{\substack{1 \leq u_{1}<u_{2}<u_{3} \leq 2 p \\ u_{1}, u_{2}, u_{3}, u_{2}-u_{1}, u_{3}-u_{2} \in \mathcal{P}_{p}}} \frac{1}{u_{1} u_{2} u_{3}}+\sum_{\substack{1 \leq u_{1}<p<u_{3} \leq 2 p \\ u_{1}, u_{3} \in \mathcal{P} p}} \frac{1}{u_{1} p u_{3}} .
$$

By Lemma 4, we have

$$
\sum_{\substack{1 \leq u_{1}<p<u_{3} \leq 2 p \\ u_{1}, u_{3} \in \mathcal{P} p}} \frac{1}{u_{1} p u_{3}}=\frac{1}{p} \sum_{1 \leq u_{1}<p} \frac{1}{u_{1}} \sum_{p<u_{3}<2 p} \frac{1}{u_{3}} \equiv 0\left(\bmod p^{3}\right) .
$$

Hence 


$$
\begin{aligned}
& \sum_{\substack{1 \leq u_{1}<u_{u}<u_{3} \leq 2 p \\
u_{1}, u_{3}, u_{2}-u_{1}, u_{3}-u_{2} \in \mathcal{P}_{p}}} \frac{1}{u_{1} u_{2} u_{3}} \equiv \sum_{\substack{1 \leq u_{1}<u_{2}<u_{3} \leq 2 p \\
u_{1}, u_{2}, u_{3}, u_{2}-u_{1}, u_{3}-u_{2} \in \mathcal{P}_{p}}} \frac{1}{u_{1} u_{2} u_{3}} \\
& \equiv \sum_{\substack{1 \leq u_{1}<u_{2}<u_{3} \leq 2 p \\
u_{1}, u_{2}, u_{3} \in \mathcal{P}_{p}}} \frac{1}{u_{1} u_{2} u_{3}}-\sum_{\substack{1 \leq u_{1}<u_{1}+p<u_{3} \leq 2 p \\
u_{1}, u_{3} \in \mathcal{P}_{p}}} \frac{1}{u_{1}\left(u_{1}+p\right) u_{3}} \\
& -\sum_{\substack{1 \leq u_{1}<u_{2}<u_{2}+p \leq p \\
u_{1}, u_{2} \in \mathcal{P}_{p}}} \frac{1}{u_{1} u_{2}\left(u_{2}+p\right)}\left(\bmod p^{3}\right) .
\end{aligned}
$$

Replace $u_{3}=u_{2}+p$, then

$$
\begin{aligned}
& \sum_{\substack{1 \leq u_{1}<u_{1}+p<u_{3} \leq 2 p \\
u_{1}, u_{3} \in \mathcal{P}_{p}}} \frac{1}{u_{1}\left(u_{1}+p\right) u_{3}}=\sum_{\substack{1 \leq u_{1}<u_{1}+p<l_{2}+p \leq 2 p \\
u_{1}, u_{2} \in \mathcal{P}_{p}}} \frac{1}{u_{1}\left(u_{1}+p\right)\left(u_{2}+p\right)} \\
& \equiv \sum_{1 \leq u_{1}<u_{2}<p} \frac{1}{u_{1}^{2} u_{2}}\left(1-\frac{p}{u_{1}}+\frac{p^{2}}{u_{1}^{2}}\right)\left(1-\frac{p}{u_{2}}+\frac{p^{2}}{u_{2}^{2}}\right)\left(\bmod p^{3}\right) .
\end{aligned}
$$

and

$$
\sum_{\substack{1 \leq u_{1}<u_{2}<u_{2}+p \leq 2 p \\ u_{1}, u_{2} \in \mathcal{P}_{p}}} \frac{1}{u_{1} u_{2}\left(u_{2}+p\right)} \equiv \sum_{\substack{1 \leq u_{1}<u_{2}<p \\ u_{1} u_{2}^{2}}} \frac{1}{u_{2}}\left(1-\frac{p}{u_{2}}+\frac{p^{2}}{u_{2}^{2}}\right)\left(\bmod p^{3}\right) .
$$

Thus

$$
\begin{aligned}
& \sum_{\substack{1 \leq u_{1}<u_{2}<u_{3} \leq 2 p \\
u_{1}, u_{3}, u_{2}-u_{1}, u_{3}-u_{2} \in \mathcal{P}_{p}}} \frac{1}{u_{1} u_{2} u_{3}} \\
\equiv & \sum_{\substack{1 \leq u_{1}<u_{2}<u_{3} \leq 2 p \\
u_{1}, u_{2}, u_{3} \in \mathcal{P}_{p}}} \frac{1}{u_{1} u_{2} u_{3}}-\sum_{1 \leq u_{1}<u_{2}<p}\left(\frac{1}{u_{1}^{2} u_{2}}+\frac{1}{u_{1} u_{2}^{2}}-p\left(\frac{1}{u_{1}^{3} u_{2}}+\frac{1}{u_{1}^{2} u_{2}^{2}}+\frac{1}{u_{1} u_{2}^{3}}\right)\right. \\
& +p^{2}\left(\frac{1}{u_{1}^{4} u_{2}}+\frac{1}{u_{1}^{3} u_{2}}+\frac{1}{u_{1}^{2} u_{2}^{3}}+\frac{1}{u_{1}^{1} u_{2}^{4}}\right) \\
\equiv & \frac{1}{3 !} \sum_{\substack{1 \leq u_{1}, u_{2}, u_{3} \leq 2 p \\
u_{i} \neq u_{j}, u_{i} \in \mathcal{P}_{p}}} \frac{1}{u_{1} u_{2} u_{3}}-\sum_{1 \leq u_{1}, u_{2}<p}\left(\frac{1}{u_{1}^{2} u_{2}}-p\left(\frac{1}{u_{1}^{3} u_{2}}+\frac{1}{2} \frac{1}{u_{1}^{2} u_{2}^{2}}\right)\right. \\
& \left.+p^{2}\left(\frac{1}{u_{1}^{4} u_{2}}+\frac{1}{u_{1}^{3} u_{2}}\right)\right)\left(\bmod p^{3}\right) .
\end{aligned}
$$

Using Lemma 5 in the first sum of the right hand in (7) and using Lemma 4 in the second sum, we have

$$
\begin{aligned}
\sum_{\substack{1 \leq u_{1}<u_{1}<u_{u} \leq 2 p \\
u_{1}, u_{3}, u_{2}-u_{1}, u_{3}-u_{2} \in \mathcal{P}_{p}}} \frac{1}{u_{1} u_{2} u_{3}} \equiv & \frac{1}{3 !}(-1)^{3}(3-1) ! \frac{24}{5} B_{p-5} p^{2}-(-1)^{2} \frac{12}{10} B_{p-5} p^{2} \\
& +\frac{3 p}{2}\left(-\frac{4}{5} B_{p-5} p\right)-p^{2} \frac{30}{14} B_{p-7} p^{2} \\
& \equiv-\frac{20}{5} p^{2} B_{p-5}\left(\bmod p^{3}\right) .
\end{aligned}
$$

Combining (5) with (8), we complete the proof of Lemma 6 .

Lemma 7. Let $p>4$ be a prime and $r>1$ a positive integer, then 


$$
\sum_{\substack{i_{1}+i_{2}+i_{3}+i_{4}=2 p^{r} \\ i_{1}, i_{2}, i_{3}, i_{4} \in \mathcal{P}_{p}}} \frac{1}{i_{1} i_{2} i_{3} i_{4}} \equiv-\frac{48}{5} p^{r} B_{p-5}\left(\bmod p^{r+1}\right) .
$$

Proof. The proof of Lemma 7 is similar to the proof method of (4) in [6].

Lemma 8 ([7]). Let $p>5$ be a prime and $r, m$ positive integers, $(m, p)=1$, then

$$
\sum_{\substack{i_{1}+i_{2}+\cdots+i_{5}=m p \\
i_{1}, i_{2}, \cdots, i_{5} \in \mathcal{P}_{p}}} \frac{1}{i_{1} i_{2} i_{3} i_{4} i_{5}} \equiv\left\{\begin{array}{l}
-4\left(5 m+m^{3}\right) B_{p-5}(\bmod p), \text { if } r=1, \\
-20 m p^{r-1} B_{p-5}\left(\bmod p^{r}\right), \text { if } r>1
\end{array}\right.
$$

Lemma 9. Let $p>5$ be a prime and $r, m$ positive integers, then

$$
\sum_{\substack{i_{1}+i_{2}+\cdots+i_{5}=m p^{r} \\ i_{1}, i_{2}, \cdots, i_{5} \in \mathcal{P}_{p}}} \frac{1}{i_{1} i_{2} i_{3} i_{4} i_{5}}=\frac{120}{m p^{r}} \sum_{\substack{1 \leq u_{1}<u_{2}<u_{3}<u_{4} \leq m p^{r} \\ u_{1}, u_{4}, u_{2}-u_{1}, u_{3}-u_{2}, u_{4}-u_{3} \in \mathcal{P}_{p}}} \frac{1}{u_{1} u_{2} u_{3} u_{4}} .
$$

Proof. The proof of Lemma 9 is similar to the proof of Lemma 2.

\section{Proofs of the Theorems}

Proof of Theorem 1. It is easy to see that

$$
\begin{aligned}
\sum_{\substack{i+j+k=2 p^{r} \\
i, j, k \in \mathcal{P}_{p}}} \frac{(-1)^{i}}{i j k} & =\frac{1}{2 p^{r}} \sum_{\substack{i+j+k=2 p^{r} \\
i, j, k \in \mathcal{P}_{p}}} \frac{(-1)^{i}(i+j+k)}{i j k} \\
& =\frac{1}{2 p^{r}} \sum_{\substack{i+j+k=2 p^{r} \\
i, j, k \in \mathcal{P}_{p}}}\left(\frac{(-1)^{i}}{j k}+\frac{2(-1)^{i}}{i j}\right) .
\end{aligned}
$$

Let $l=j+k$,then $1 \leq j<l \leq 2 p^{r}$ and $j, l, l-j \in \mathcal{P}_{p}$, hence

$$
\sum_{\substack{i+j+k=2 p^{r} \\ i, j, k \in \mathcal{P}_{p}}} \frac{(-1)^{i}}{j k}=\sum_{\substack{1 \leq j<l \leq 2 p^{r} \\ j, l, l-j \in \mathcal{P}_{p}}} \frac{1}{l} \frac{(-1)^{l}(j+k)}{j k}=\sum_{\substack{1 \leq j<l \leq 2 p^{r} \\ j, l, l-j \in \mathcal{P}_{p}}} \frac{2(-1)^{l}}{j l} .
$$

Let $l^{\prime}=i+j$, then $1 \leq i<l^{\prime} \leq 2 p^{r}$ and $i, l^{\prime}, l^{\prime}-i \in \mathcal{P}_{p}$, hence

$$
\sum_{\substack{i+j+k=2 p^{r} \\ i, j, k \in \mathcal{P}_{p}}} \frac{2(-1)^{i}}{i j}=\sum_{\substack{1 \leq i<l^{\prime} \leq 2 p^{r} \\ i, l^{\prime}, l^{\prime}-i \in \mathcal{P}_{p}}} \frac{1}{l^{\prime}} \frac{2(-1)^{i}(i+j)}{i j}=\sum_{\substack{1 \leq i<l^{\prime} \leq 2 p^{r} \\ i, l^{\prime}, l^{\prime}-i \in \mathcal{P}_{p}}}\left(\frac{2(-1)^{i}}{j l^{\prime}}+\frac{2(-1)^{i}}{i l^{\prime}}\right) .
$$

Noting that $i=l^{\prime}-j,(-1)^{l^{\prime}-j}=(-1)^{l^{\prime}+j}$ and we rename $l^{\prime}$ to $l$, then

$$
\sum_{\substack{1 \leq i<l^{\prime} \leq 2 p^{r} \\ i, l^{\prime}, l^{\prime}-i \in \mathcal{P}_{p}}} \frac{2(-1)^{i}}{j l^{\prime}}=\sum_{\substack{1 \leq j<l \leq 2 p^{r} \\ j, l, l-j \in \mathcal{P}_{p}}} \frac{2(-1)^{j+l}}{j l} .
$$

Rename $i$ to $j$ and $l^{\prime}$ to $l$, then

$$
\sum_{\substack{1 \leq i<l^{\prime} \leq 2 p^{r} \\ i, l^{\prime}, l^{\prime}-i \in \mathcal{P}_{p}}} \frac{2(-1)^{i}}{i l^{\prime}}=\sum_{\substack{1 \leq j<l \leq 2 p^{r} \\ j, l, l-j \in \mathcal{P}_{p}}} \frac{2(-1)^{j}}{j l}
$$

Combining (9)-(13), we have 


$$
\begin{aligned}
\sum_{\substack{i+j+k=2 p^{r} \\
i, j, k \in \mathcal{P}_{p}}} \frac{(-1)^{i}}{i j k} & =\frac{1}{p^{r}} \sum_{\substack{1 \leq j<l \leq 2 p^{r} \\
j, l, l-j \in \mathcal{P}_{p}}}\left(\frac{(-1)^{l}}{j l}+\frac{(-1)^{j+l}}{j l}+\frac{(-1)^{j}}{j l}\right) \\
& =\frac{1}{p^{r}} \sum_{\substack{1 \leq j<l<2 p^{r} \\
j, l, l-j \in \mathcal{P}_{p}}} \frac{\left(1+(-1)^{l}\right)\left(1+(-1)^{j}\right)}{j l}-\frac{1}{p^{r}} \sum_{\substack{1 \leq j<l \leq 2 p^{r} \\
j, l, l-j \in \mathcal{P}_{p}}} \frac{1}{j l} \\
& =\frac{1}{p^{r}} \sum_{\substack{1 \leq j<l \leq 2 p^{r} \\
j, l, l-j \in \mathcal{P}_{p}, j \text { even, }, \text { even }}} \frac{4}{j l}-\frac{1}{p^{r}} \sum_{\substack{1 \leq j<l \leq 2 p^{r} \\
j, l, l-j \in \mathcal{P}_{p}}} \frac{1}{j l} .
\end{aligned}
$$

Let $j=2 j^{\prime}, l=2 l^{\prime}$ in the first sum of (14) and noting that

$$
\sum_{\substack{1 \leq j^{\prime}<l^{\prime} \leq p^{r} \\ j^{\prime}, l^{\prime}, l^{\prime}-j^{\prime} \in \mathcal{P}_{p}}} \frac{1}{j^{\prime} l^{\prime}}=\sum_{\substack{1 \leq j<l \leq p^{r} \\ j, l, l-j \in \mathcal{P}_{p}}} \frac{1}{j l},
$$

(14) is equal to

$$
\sum_{\substack{i+j+k=2 p^{r} \\ i, j, k \in \mathcal{P}_{p}}} \frac{(-1)^{i}}{i j k}=\frac{1}{p^{r}} \sum_{\substack{1 \leq j<l \leq p^{r} \\ j, l, l-j \in \mathcal{P}_{p}}} \frac{1}{j l}-\frac{1}{p^{r}} \sum_{\substack{1 \leq j<l \leq 2 p^{r} \\ j, l, l-j \in \mathcal{P}_{p}}} \frac{1}{j l} .
$$

By Lemma 1, Lemma 2 and (15), we obtain

$$
\sum_{\substack{i+j+k=2 p^{r} \\ i, j, k \in \mathcal{P}_{p}}} \frac{(-1)^{i}}{i j k}=\frac{1}{p^{r}} \sum_{\substack{1 \leq j<l \leq p^{r} \\ j, l, l-j \in \mathcal{P}_{p}}} \frac{1}{j l}-\frac{1}{p^{r}} \sum_{\substack{1 \leq j<l \leq 2 p^{r} \\ j, l, l-j \in \mathcal{P}_{p}}} \frac{1}{j l} \equiv p^{r-1} B_{p-3}\left(\bmod p^{r}\right) .
$$

This completes the proof of Theorem 1 .

Proof of Theorem 2. For every triple $(i, j, k)$ of positive integers which satisfies $i+j+k=2 p^{r}, i, j, k \in \mathcal{P}_{p}$, we take it to 3 cases.

Cases 1. Let $A\left(p^{r}\right)=\left\{(i, j, k) \mid 1 \leq i, j, k \leq p^{r}-1\right.$ and $\left.i, j, k \in \mathcal{P}_{p}\right\}$.

$(i, j, k) \leftrightarrow\left(p^{r}-i, p^{r}-j, p^{r}-k\right)$ is a bijection between the solutions of $i+j+k=2 p^{r},(i, j, k) \in A\left(p^{r}\right)$ and $i+j+k=p^{r}, i, j, k \in \mathcal{P}_{p}$, we have

$$
\begin{aligned}
\sum_{\substack{i+j+k=2 p^{r} \\
(i, j, k) \in A\left(p^{r}\right)}} \frac{(-1)^{i}}{i j k} & \equiv \sum_{\substack{i+j+k=p^{r} \\
i, j, k \in \mathcal{P}_{p}}} \frac{(-1)^{p^{r}-i}}{\left(p^{r}-i\right)\left(p^{r}-j\right)\left(p^{r}-k\right)} \\
& \equiv \sum_{\substack{i+j+k=p^{r} \\
i, j, k \in \mathcal{P}_{p}}} \frac{(-1)^{i}}{i j k}\left(\bmod p^{r}\right) .
\end{aligned}
$$

Cases 2. Let

$B\left(p^{r}\right)=\left\{(i, j, k) \mid p^{r}+1 \leq i \leq 2 p^{r}-1,1 \leq j, k \leq p^{r}-1\right.$ and $\left.i, j, k \in \mathcal{P}_{p}\right\}$. $(i, j, k) \leftrightarrow\left(p^{r}+i, j, k\right)$ is a bijection between the solutions of $i+j+k=2 p^{r},(i, j, k) \in B\left(p^{r}\right)$ and $i+j+k=p^{r}, i, j, k \in \mathcal{P}_{p}$, we have

$$
\sum_{\substack{i+j+k=2 p^{r} \\(i, j, k) \in B\left(p^{r}\right)}} \frac{(-1)^{i}}{i j k} \equiv \sum_{\substack{i+j+k=p^{r} \\ i, j, k \in \mathcal{P}_{p}}} \frac{(-1)^{p^{r}+i}}{\left(p^{r}+i\right) j k} \equiv-\sum_{\substack{i+j+k=p^{r} \\ i, j, k \in \mathcal{P}_{p}}} \frac{(-1)^{i}}{i j k}\left(\bmod p^{r}\right) .
$$

Cases 3. Let

$$
C\left(p^{r}\right)=\left\{(i, j, k) \mid p^{r}+1 \leq j \leq 2 p^{r}-1,1 \leq i, k \leq p^{r}-1 \text { and } i, j, k \in \mathcal{P}_{p}\right\}
$$


and

$$
D\left(p^{r}\right)=\left\{(i, j, k) \mid p^{r}+1 \leq k \leq 2 p^{r}-1,1 \leq i, j \leq p^{r}-1 \text { and } i, j, k \in \mathcal{P}_{p}\right\} .
$$

$(i, j, k) \leftrightarrow\left(i, p^{r}+j, k\right)$ in the former and $(i, j, k) \leftrightarrow\left(i, j, p^{r}+k\right)$ in the later are the bijections between the solutions of $i+j+k=2 p^{r},(i, j, k) \in C\left(p^{r}\right)$ or $(i, j, k) \in D\left(p^{r}\right)$ and $i+j+k=p^{r}, i, j, k \in \mathcal{P}_{p}$, we have

$$
\begin{aligned}
& \sum_{\substack{i+j+k=2 p^{r} \\
(i, j, k) \in C\left(p^{r}\right)}} \frac{(-1)^{i}}{i j k}+\sum_{\substack{i+j+k=2 p^{r} \\
(i, j, k) \in D\left(p^{r}\right)}} \frac{(-1)^{i}}{i j k} \\
\equiv & \sum_{\substack{i+j+k=p^{r} \\
i, j, k \in \mathcal{P}_{p}}} \frac{(-1)^{i}}{i\left(p^{r}+j\right) k}+\sum_{\substack{i+j+k=p^{r} \\
i, j, k \in \mathcal{P}_{p}}} \frac{(-1)^{i}}{i j\left(p^{r}+k\right)} \\
\equiv & 2 \sum_{\substack{i+j+k=p^{r} \\
i, j, k \in \mathcal{P}_{p}}} \frac{(-1)^{i}}{i j k}\left(\bmod p^{r}\right) .
\end{aligned}
$$

Combining (16)-(18), we have

$$
\begin{aligned}
\sum_{\substack{i+j+k=2 p^{r} \\
i, j, k \in \mathcal{P}_{p}}} \frac{(-1)^{i}}{i j k}=\sum_{\substack{i+j+k=2 p^{r} \\
(i, j, k) \in A\left(p^{r}\right)}} \frac{(-1)^{i}}{i j k}+\sum_{\substack{i+j+k=2 p^{r} \\
(i, j, k) \in B\left(p^{r}\right)}} \frac{(-1)^{i}}{i j k} \\
+\sum_{\substack{i+j+k=2 p^{r} \\
(i, j, k) \in C\left(p^{r}\right)}} \frac{(-1)^{i}}{i j k}+\sum_{\substack{i+j+k=2 p^{r} \\
(i, j, k) \in D\left(p^{r}\right)}} \frac{(-1)^{i}}{i j k} \\
\equiv 2 \sum_{\substack{i+j+k=p^{r} \\
i, j, k \in \mathcal{P}_{p}}} \frac{(-1)^{i}}{i j k}\left(\bmod p^{r}\right) .
\end{aligned}
$$

By Theorem 1, we complete the proof of Theorem 2.

Proof of Theorem 3. By symmetry, it is easy to see that

$$
\begin{aligned}
\sum_{\substack{i_{1}+i_{2}+i_{3}+i_{4}=2 p^{r} \\
i_{1}, i_{2}, i_{3}, i_{4} \in \mathcal{P}_{p}}} \frac{(-1)^{r}}{i_{1} i_{2} i_{3} i_{4}} & =\frac{1}{2 p^{r}} \sum_{\substack{i_{1}+i_{2}+i_{3}+i_{4}=2 p^{r} \\
i_{1}, i_{2}, i_{3}, i_{4} \in \mathcal{P}_{p}}} \frac{(-1)^{i_{1}}\left(i_{1}+i_{2}+i_{3}+i_{4}\right)}{i_{1} i_{2} i_{3} i_{4}} \\
& =\frac{1}{2 p^{r}} \sum_{\substack{i_{1}+i_{2}+i_{3}+i_{4}=2 p^{r} \\
i_{1}, i_{2}, i_{3}, i_{4} \in \mathcal{P}_{p}}}\left(\frac{(-1)^{i_{1}}}{i_{2} i_{3} i_{4}}+3 \frac{(-1)^{i_{1}}}{i_{1} i_{3} i_{4}}\right) .
\end{aligned}
$$

Let $u_{3}=i_{2}+i_{3}+i_{4}$ in the first sum of the last equation in (19), then $i_{1}=2 p^{r}-u_{3}$, (19) equals to

$$
\begin{aligned}
& =\frac{1}{2 p^{r}}\left[\sum_{\substack{u_{3}=i_{2}+i_{i}+i_{i}<2 p^{r} \\
u_{3}, i_{2}, i_{3}, i_{4} \in \mathcal{P}_{p}}} \frac{(-1)^{2 p^{r}-u_{3}\left(i_{2}+i_{3}+i_{4}\right)}}{i_{2} i_{3} i_{4} u_{3}}+3 \sum_{\substack{u_{3}=i_{1}+i_{3}+i_{4}<2 p^{r} \\
u_{3}, i_{1}, i_{3}, i_{4} \in \mathcal{P}_{p}}} \frac{(-1)^{i_{1}}\left(i_{1}+i_{3}+i_{4}\right)}{i_{1} i_{3} i_{4} u_{3}}\right] \\
& =\frac{1}{2 p^{r}}\left[3 \sum_{\substack{u_{3}=i_{2}+i_{3}+i_{4}<2 p^{r} \\
u_{3}, i_{2}, i_{3}, i_{4} \in \mathcal{P}_{p}}} \frac{(-1)^{u_{3}}}{i_{3} i_{4} u_{3}}+3 \sum_{\substack{u_{3}=i_{1}+i_{3}+i_{4}<2 p^{r} \\
u_{3}, i_{1}, i_{3}, i_{4} \in \mathcal{P}_{p}}} \frac{(-1)^{r}}{i_{3} i_{4} u_{3}}+6 \sum_{\substack{i_{3}=i_{1}+i_{3}+i_{4}<2 p^{r} \\
u_{3}, i_{1}, i_{3}, i_{4} \in \mathcal{P}_{p}}} \frac{(-1)^{i_{1}}}{i_{1} i_{3} u_{3}}\right] .
\end{aligned}
$$


Let $u_{2}=i_{3}+i_{4}$ in the second sum of the last equation in (20), since $u_{3}=i_{1}+i_{3}+i_{4}$, then $i_{1}=u_{3}-u_{2}$, (20) equals to

$$
\begin{aligned}
& =\frac{1}{2 p^{r}}\left[3 \sum_{\substack{u_{1}=i_{i}+i_{4}<u_{3}<2 p^{r} \\
u_{3}, u_{3}-u_{2}, i_{3}, i_{4} \in \mathcal{P}_{p}}} \frac{(-1)^{u_{3}}\left(i_{3}+i_{4}\right)}{i_{3} i_{4} u_{2} u_{3}}+3 \sum_{\substack{u_{2}=i_{3} i_{i}<u_{3}<2 p^{r} \\
u_{3}, u_{3}-u_{2}, i_{3}, i_{4} \in \mathcal{P}_{p}}} \frac{(-1)^{u_{3}-u_{2}}\left(i_{3}+i_{4}\right)}{i_{3} i_{4} u_{3}}\right. \\
& \left.+6 \sum_{\substack{u_{2}=i_{1}+i_{3}<u_{3}<2 p^{r} \\
u_{3}, u_{3}-u_{2}, i_{1}, i_{3} \in \mathcal{P}_{p}}} \frac{(-1)^{i_{1}}\left(i_{1}+i_{3}\right)}{i_{1} i_{3} u_{2} u_{3}}\right] \\
& =\frac{1}{2 p^{r}}\left[6 \sum_{\substack{0<u_{1}<u_{2}<u_{3}<2 p^{r} \\
u_{1}, u_{3}, u_{3}-u_{2}, u_{2}-u_{1} \in \mathcal{P}_{p}}} \frac{(-1)^{u_{3}}}{u_{1} u_{2} u_{3}}+6 \sum_{\substack{0<u_{1}<u_{2}<u_{3}<2 p^{r} \\
u_{1}, u_{3}, u_{3}-u_{2}, u_{2}-u_{1} \in \mathcal{P}_{p}}} \frac{(-1)^{u_{3}-u_{2}}}{u_{1} u_{2} u_{3}}\right. \\
& \left.+6 \sum_{\substack{0<u_{1}<u_{2}<u_{3}<2 p^{r} \\
u_{1}, u_{3}, u_{3}-u_{2}, u_{2}-u_{1} \in \mathcal{P}_{p}}} \frac{(-1)^{u_{2}-u_{1}}}{u_{1} u_{2} u_{3}}+6 \sum_{\substack{0<u_{1}<u_{2}<u_{3}<2 p^{r} \\
u_{1}, u_{3}, u_{3}-u_{2}, u_{2}-u_{1} \in \mathcal{P}_{p}}} \frac{(-1)^{u_{1}}}{u_{1} u_{2} u_{3}}\right] \\
& =\frac{3}{p^{r}} \sum_{\substack{0<u_{1}<u_{2}<u_{3}<2 p^{r} \\
u_{1}, u_{3}, u_{3}-u_{2}, u_{2}-u_{1} \in \mathcal{P}_{p}}} \frac{(-1)^{u_{3}}+(-1)^{u_{2}+u_{3}}+(-1)^{u_{1}+u_{2}}+(-1)^{u_{1}}}{u_{1} u_{2} u_{3}} .
\end{aligned}
$$

Similarly, we have

$$
\sum_{\substack{i_{1}+i_{2}+i_{3}+i_{i}=2 p^{r} \\ i_{1}, i_{2}, i_{3}, i_{4} \in p_{p}}} \frac{(-1)^{i_{1}+i_{2}}}{i_{1} i_{2} i_{3} i_{4}}=\frac{4}{p^{r}} \sum_{\substack{0<u_{1}<u_{2}<u_{3}<2 p^{r} \\ u_{1}, u_{3}, u_{3}-u_{2}, u_{2}-u_{1} \in \mathcal{P}_{p}}} \frac{(-1)^{u_{2}}+(-1)^{u_{1}+u_{2}+u_{3}}+(-1)^{u_{1}+u_{3}}}{u_{1} u_{2} u_{3}} .
$$

\section{Hence}

$$
\begin{aligned}
& 4 \sum_{\substack{i_{1}+i_{2}+i_{3}+i_{4}=2 p^{2} \\
i_{1}, i_{2}, i_{3}, i_{4} \in \mathcal{P}_{p}}} \frac{(-1)^{r}}{i_{1} i_{2} i_{3} i_{4}}+3 \sum_{\substack{i_{1} \\
i_{1}+i_{2}+i_{3}+i_{4}=2 p^{r} \\
i_{1}, i_{2}, i_{3}, i_{4} \in \mathcal{P}_{p}}} \frac{(-1)^{i_{1}+i_{2}}}{i_{1} i_{2} i_{3} i_{4}} \\
& =\frac{12}{p^{r}} \sum_{\substack{0<u_{1}<u_{2}<u_{3}<2 p^{r} \\
u_{1}, u_{3}, u_{3}-u_{2}, u_{2}-u_{1} \in \mathcal{P}_{p}}} \frac{\left[1+(-1)^{u_{1}}\right]\left[1+(-1)^{u_{2}}\right]\left[1+(-1)^{u_{3}}\right]-1}{u_{1} u_{2} u_{3}} \\
& =\frac{12}{p^{r}} \sum_{\substack{0<u_{1}<u_{2}<u_{3}<p^{r} \\
u_{1}, u_{3}, u_{3}-u_{2}, u_{2}-u_{1} \in \mathcal{P}_{p}}} \frac{1}{u_{1} u_{2} u_{3}}-\frac{12}{p^{r}} \sum_{\substack{0<u_{1}<u_{2}<u_{3}<2 p^{r} \\
u_{1}, u_{3}, u_{3}-u_{2}, u_{2}-u_{1} \in \mathcal{P}_{p}}} \frac{1}{u_{1} u_{2} u_{3}} .
\end{aligned}
$$

By Lemma 3, we have

$$
\begin{aligned}
& 4 \sum_{\substack{i_{1}+i_{2}+i_{3}+i_{4}=2 p^{r} \\
i_{1}, i_{2}, i_{3}, i_{4} \in \mathcal{P}_{p}}} \frac{(-1)^{i_{1}}}{i_{1} i_{2} i_{3} i_{4}}+3 \sum_{\substack{i_{3} \\
i_{1}+i_{2}+i_{3}+i_{4}=2 p^{r} \\
i_{1}, i_{2}, i_{3}, i_{4} \in \mathcal{P}_{p}}} \frac{(-1)^{i_{1}+i_{2}}}{i_{1} i_{2} i_{3} i_{4}} \\
& =\frac{1}{2} \sum_{\substack{i_{1}+i_{2}+i_{i}+i_{4}=2 p^{r} \\
i_{1}, i_{2}, i_{3}, i_{4} \in \mathcal{P}_{p}}} \frac{1}{i_{1} i_{2} i_{3} i_{3} i_{4}}-\sum_{\substack{i_{1}+i_{2}+i_{i}+i_{4}=2 p^{r} \\
i_{1}, i_{2}, i_{3}, i_{4} \in \mathcal{P} p}} \frac{1}{i_{1} i_{2} i_{3} i_{4}} .
\end{aligned}
$$

By (2) and Lemma 6, we have

$$
\begin{aligned}
& 4 \sum_{\substack{i_{1}+i_{2}+i_{3}+i_{4}=2 p \\
i_{1}, i_{2}, i_{3}, i_{4} \in \mathcal{P}_{p}}} \frac{(-1)^{i_{1}}}{i_{1} i_{2} i_{3} i_{4}}+3 \sum_{\substack{i_{3} \\
i_{1}+i_{2}+i_{i}+i_{4}=2 p \\
i_{1}, i_{2}, i_{3}, i_{4} \in \mathcal{P}_{p}}} \frac{(-1)^{i_{1}+i_{2}}}{i_{1} i_{2} i_{3} i_{3} i_{4}} \\
& \equiv-\frac{24}{5} p B_{p-5}+\frac{240}{5} p B_{p-5} \equiv \frac{216}{5} p B_{p-5}\left(\bmod p^{2}\right) .
\end{aligned}
$$


By (4) and Lemma 7, if $r \geq 2$, then

$$
\begin{aligned}
& 4 \sum_{\substack{i_{1}+i_{2}+i_{3}+i_{i}=2 p^{r} \\
i_{1}, i_{2}, i_{3}, i_{4} \in \mathcal{P}_{p}}} \frac{(-1)^{i_{1}}}{i_{1} i_{2} i_{3} i_{4}}+3 \sum_{\substack{i_{1}+i_{2}+i_{3}+i_{4}=2 p^{r} \\
i_{1}, i_{2}, i_{3}, i_{4} \in \mathcal{P}_{p}}} \frac{(-1)^{i_{1}+i_{2}}}{i_{1} i_{2} i_{3} i_{4}} \\
& \equiv-\frac{12}{5} p^{r} B_{p-5}+\frac{48}{5} p^{r} B_{p-5} \equiv \frac{36}{5} p^{r} B_{p-5}\left(\bmod p^{r+1}\right) .
\end{aligned}
$$

This completes the proof of Theorem 3 .

Proof of Theorem 4. Similar to the proofs of Theorem 1 and Theorem 3, we have

$$
\begin{aligned}
\sum_{\substack{i_{1}+i_{2}+i_{3}+i_{4}+i_{5}=2 p^{r} \\
i_{1}, i_{2}, i_{3}, i_{4}, i_{5} \in \mathcal{P}_{p}}} \frac{(-1)^{i_{1}}}{i_{1} i_{2} i_{3} i_{4} i_{5}}= & \frac{12}{p^{r}} \sum_{\substack{0<u_{1}<u_{2}<u_{3}<u_{4}<2 p^{r} \\
u_{1}, u_{4}, u_{2}-u_{1}, u_{3}-u_{2}, u_{4}-u_{3} \in \mathcal{P}_{p}}} \frac{1}{u_{1} u_{2} u_{3} u_{4}}\left[(-1)^{u_{1}}\right. \\
& \left.\left.+(-1)^{u_{4}}+(-1)^{u_{1}+u_{2}}+(-1)\right)^{u_{2}+u_{3}}+(-1)^{u_{3}+u_{4}}\right]
\end{aligned}
$$

and

$$
\begin{aligned}
& \sum_{\substack{i_{1}+i_{2}+i_{3}+i_{4}+i_{5}=2 p^{r_{1}} \\
i_{1}, i_{2}, i_{3}, i_{4}, i_{5} \in \mathcal{P}_{p}}} \frac{(-1)^{i_{1}+i_{2}}}{i_{1} i_{2} i_{3} i_{4} i_{5}} \\
&= \frac{6}{p^{r}} \sum_{\substack{0<u_{1}<u_{2}<u_{3}<u_{4}<2 p^{r} \\
u_{1}, u_{4}, u_{2}-u_{1}, u_{3}-u_{2}, u_{4}-u_{3} \in \mathcal{P}_{p}}} \frac{1}{u_{1} u_{2} u_{3} u_{4}}\left[(-1)^{u_{2}}+(-1)^{u_{3}}\right. \\
&+(-1)^{u_{1}+u_{3}}+(-1)^{u_{1}+u_{4}}+(-1)^{u_{2}+u_{4}}+(-1)^{u_{1}+u_{2}+u_{3}} \\
&\left.+(-1)^{u_{1}+u_{2}+u_{4}}+(-1)^{u_{1}+u_{3}+u_{4}}+(-1)^{u_{2}+u_{3}+u_{4}}+(-1)^{u_{1}+u_{2}+u_{3}+u_{4}}\right]
\end{aligned}
$$

Hence

$$
\begin{aligned}
& \sum_{\substack{i_{1}+i_{2}+i_{3}+i_{4}+i_{5}=2 p^{r} \\
i_{1}, i_{2}, i_{3}, i_{4}, i_{5} \in \mathcal{P}_{p}}} \frac{(-1)^{i_{1}}}{i_{1} i_{2} i_{3} i_{4} i_{5}}+2 \sum_{\substack{i_{1}+i_{2}+i_{3}+i_{4}+i_{5}=2 p^{r} \\
i_{1}, i_{2}, i_{3}, i_{4}, i_{5} \in \mathcal{P}_{p}}} \frac{(-1)^{i_{1}+i_{2}}}{i_{1} i_{2} i_{3} i_{4} i_{5}} \\
& =\frac{12}{p^{r}} \sum_{\substack{0<u_{1}<u_{2}<u_{3}<u_{4}<2 p^{r} \\
u_{1}, u_{4}, u_{2}-u_{1}, u_{3}-u_{2}, u_{4}-u_{3} \in \mathcal{P}_{p}}} \frac{\left[1+(-1)^{u_{1}}\right]\left[1+(-1)^{u_{2}}\right]\left[1+(-1)^{u_{3}}\right]\left[1+(-1)^{u_{4}}\right]-1}{u_{1} u_{2} u_{3} u_{4}} \\
& =\frac{12}{p^{r}} \sum_{\substack{0<u_{1}<u_{2}<u_{3}<u_{4}<p^{r} \\
u_{1}, u_{4}, u_{2}-u_{1}, u_{3}-u_{2}, u_{4}-u_{3} \in \mathcal{P}_{p}}} \frac{1}{u_{1} u_{2} u_{3} u_{4}}-\frac{12}{p^{r}} \sum_{\substack{0<u_{1}<u_{2}<u_{3}<u_{4}<2 p^{r} \\
u_{1}, u_{4}, u_{2}-u_{1}, u_{3}-u_{2}, u_{4}-u_{3} \in \mathcal{P}_{p}}} \frac{1}{u_{1} u_{2} u_{3} u_{4}} .
\end{aligned}
$$

By Lemma 9, we have

$$
\begin{aligned}
& \sum_{\substack{i_{1}+i_{2}+i_{3}+i_{4}+i_{5}=2 p^{r} \\
i_{1}, i_{2}, i_{3}, i_{4}, i_{5} \in \mathcal{P}_{p}}} \frac{(-1)^{i_{1}}}{i_{1} i_{2} i_{3} i_{4} i_{5}}+2 \sum_{\substack{i_{1}+i_{2}+i_{3}+i_{4}+i_{5}=2 p^{r} \\
i_{1}, i_{2}, i_{3}, i_{4}, i_{5} \in \mathcal{P}}} \frac{(-1)^{i_{1}+i_{2}}}{i_{1} i_{2} i_{3} i_{3} i_{5}} \\
& =\frac{1}{10} \sum_{\substack{i_{1}+i_{2}+i_{3}+i_{4}+i_{5}=p^{r} \\
i_{1}, i_{2}, i_{3}, i_{4}, i_{5} \in \mathcal{P}_{p}}} \frac{1}{i_{1} i_{2} i_{3} i_{4} i_{5}}-\frac{2}{10} \sum_{\substack{i_{1}+i_{2}+i_{3}+i_{4}+i_{5}=2 p^{r} \\
i_{1}, i_{2}, i_{3}, i_{4}, i_{5} \in \mathcal{P}_{p}}} \frac{1}{i_{1} i_{2} i_{3} i_{4} i_{5}} .
\end{aligned}
$$

By (2) and Lemma 8 (1), we have 


$$
\begin{aligned}
& \sum_{\substack{i_{1}+i_{2}+i_{3}+i_{4}+i_{i}=2 p \\
i_{1}, i_{2}, i_{3}, i_{4}, i_{5} \in \mathcal{P}_{p}}} \frac{(-1)^{i_{1}}}{i_{1} i_{2} i_{3} i_{4} i_{5}}+2 \sum_{\substack{i_{1} \\
i_{1}+i_{i}+i_{3}+i_{4}+i_{5}=2 p \\
i_{1}, i_{2}, i_{3}, i_{4}, i_{5} \in \mathcal{P}_{p}}} \frac{(-1)^{i_{1}+i_{2}}}{i_{1} i_{2} i_{3} i_{4} i_{4} i_{5}} \\
& \equiv-\frac{24}{10} B_{p-5}+\frac{144}{10} B_{p-5} \equiv 12 B_{p-5}(\bmod p) .
\end{aligned}
$$

By Lemma 8 (2), if $r \geq 2$, then

$$
\begin{gathered}
\sum_{\substack{i_{1}+i_{i}+i_{3}+i_{4}+i_{5}=2 p^{r} \\
i_{1}, i_{2}, i_{3}, i_{4}, i_{5} \in \mathcal{P}_{p}}} \frac{(-1)^{i_{1}}}{i_{1} i_{2} i_{3} i_{4} i_{5}}+2 \sum_{\substack{i_{1}+i_{2}+i_{3}+i_{4}+i_{5}=2 p^{r} \\
i_{1}, i_{2}, i_{3}, i_{4}, i_{5} \in \mathcal{P}_{p}}} \frac{(-1)^{i_{1}+i_{2}}}{i_{1} i_{2} i_{3} i_{3} i_{4} i_{5}} \\
\equiv-2 p^{r-1} B_{p-5}+8 p^{r-1} B_{p-5} \equiv 6 p^{r-1} B_{p-5}\left(\bmod p^{r}\right) .
\end{gathered}
$$

This completes the proof of Theorem 4 .

\section{Conclusions}

Let $p$ be an odd prime and $r, m$ positive integers, $(m, p)=1$, using Lemma 1 and Lemma 2, similar to the proof of Theorem 1, we can prove that

$$
\sum_{\substack{i+j+k=2 m p^{r} \\ i, j, k \in \mathcal{P}_{p}}} \frac{(-1)^{i}}{i j k} \equiv m p^{r-1} B_{p-3}\left(\bmod p^{r}\right) .
$$

In particular, if $m=1$, it becomes Theorem 1 .

Let $p$ be odd prime and $r, m$ positive integers, $(m, p)=1$, similar to the proof of Theorem 2, we can prove that

$$
\sum_{\substack{i+j+k=m p r^{r} \\ i, j, k \in \mathcal{P}_{p}}} \frac{(-1)^{i}}{i j k} \equiv \frac{1}{2} \sum_{\substack{i+j+k=2 m p \\ i, j, k \in \mathcal{P}_{p}}} \frac{(-1)^{i}}{i j k} \equiv \frac{m}{2} p^{r-1} B_{p-3}\left(\bmod p^{r}\right) .
$$

In particular, if $m=1$, it becomes Theorem 2 .

Let $p>4$ be a prime and $r, m$ positive integers, $(m, p)=1$, we can deduce the congruence $\left(\bmod p^{r+1}\right)$ for

$$
4 \sum_{\substack{i_{1}+i_{2}+i_{3}+i_{3}=2 m r \\ i_{1}, i_{2}, i_{3}, i_{4} \in \mathcal{P}_{p}}} \frac{(-1)^{i_{1}}}{i_{1} i_{2} i_{2} i_{3} i_{4}}+3 \sum_{\substack{i_{1} \\ i_{1}+i_{2}+i_{3}+i_{3}=2 m p \\ i_{1}, i_{2}, i_{3}, i_{4} \in \mathcal{P} p}} \frac{(-1)^{i_{1}+i_{2}}}{i_{1} i_{2} i_{3} i_{3}} .
$$

Let $p>5$ be a prime and $r, m$ positive integers, $(m, p)=1$, we can deduce the congruence $\left(\bmod p^{r}\right)$ for

$$
\sum_{\substack{i_{1}+i_{2}+i_{3}+i_{i}+i_{i}=2 m p^{\prime} \\ i_{1}, i_{2}, i_{3}, i_{4}, i_{5} \in \mathcal{P}_{p}}} \frac{(-1)^{r}}{i_{1} i_{2} i_{3} i_{4} i_{5}}+2 \sum_{\substack{i_{1} \\ i_{1}+i_{2}+i_{3}+i_{4}+i_{i}=2 m p^{r} \\ i_{1}, i_{2}, i_{3}, i_{4}, i_{5} \in \mathcal{P}_{p}}} \frac{(-1)^{i_{1}+i_{2}}}{i_{1} i_{2} i_{3} i_{4} i_{5}} .
$$

Similarly, we can consider the congruence $\left(\bmod p^{r+1}\right)$ for

$$
\sum_{\substack{i_{1}+i_{2}+\cdots+i_{n}=p^{r} \\ i_{1}, i_{2}, \cdots, i_{n} \in \mathcal{P}_{p}}} \frac{\sigma_{1}^{i_{1}} \sigma_{2}^{i_{2}} \cdots \sigma_{n}^{i_{n}}}{i_{1} i_{2} \cdots i_{n}},
$$

where $\sigma_{i} \in\{1,-1\}, i=1,2, \cdots, n$, but it seems much more complicated. 


\section{Founding}

This work is supported by the Natural Science Foundation of Zhejiang Province, Project (No. LY18A010016) and the National Natural Science Foundation of China, Project (No. 12071421).

\section{Conflicts of Interest}

The authors declare no conflicts of interest regarding the publication of this paper.

\section{References}

[1] Zhao, J. (2007) Bernoulli Numbers, Wolstenholme's Theorem, and $p^{5}$ Variations of Lucas' Theorem. Journal of Number Theory, 123, 18-26. https://doi.org/10.1016/j.jnt.2006.05.005

[2] Ji, C. (2005) A Simple Proof of a Curious Congruence by Zhao. Proceedings of the American Mathematical Society, 133, 3469-3472. https://doi.org/10.1090/S0002-9939-05-07939-6

[3] Zhou, X. and Cai, T. (2007) A Generalization of a Curious Congruence on Harmonic Sums. Proceedings of the American Mathematical Society, 135, 1329-1333. https://doi.org/10.1090/S0002-9939-06-08777-6

[4] Xia, B. and Cai, T. (2010) Bernoulli Numbers and Congruences for Harmonic Sums. International Journal of Number Theory, 6, 849-855. https://doi.org/10.1142/S1793042110003265

[5] Wang, L. and Cai, T. (2014) A Curious Congruence Modulo Prime Power. Journal of Number Theory, 144, 15-24. https://doi.org/10.1016/j.jnt.2014.04.004

[6] Zhao, J. (2014) Congruences Involving Multiple Harmonic Sums and Finite Multiple Zeta Vakues. arxiv:1404.3549.

[7] Wang, L. (2015) A New Super Congruence Involving Multiple Harmonic Sums. Journal of Number Theory, 154, 16-31. https://doi.org/10.1016/j.jnt.2015.01.021

[8] Wang, L. (2014) A Curious Congruence Involving Alternating Harmonic Sums. Journal of Combinatorics and Number Theory, 6, 209-214. 